\title{
Identification of Deoxynucleoside- Polyaromatic Hydrocarbon Adducts by Capillary Zone Electrophoresis- Continuous Flow-Fast Atom Bombardment Mass Spectrometry
}

\author{
Susan M. Wolf and Paul Vouros \\ Department of Chemistry and Barnett Institute, Northeastern University, Boston, Massachusetts, USA
}

Curt Norwood and Eugene Jackim

US Environmental Protection Agency-ERLN, Narragansett, Rhode Island, USA

Capillary zone electrophoresis coupled to continuous flow-fast atom bombardment mass spectrometry is shown to have utility for the detection and characterization of adducts formed by the covalent attachment of four polyaromatic hydrocarbon (PAH) and amino-PAH compounds to deoxyguanosine. Normal scanning provided structural information for a 1.3 ng injection of a model adduct, while $1.3 \mathrm{ng}$ of each of a mixture of adducts was sufficient to determine their molecular weights by monitoring the constant neutral loss of deoxyribose. Exploitation of this loss in the multiple reaction monitoring mode resulted in the detection of low picogram amounts of target adducts in mixtures. (J Am Soc Mass Spectrom 1992, 3, 757-761)

$\mathrm{D}$ NA adducts are formed by the covalent attachment of xenobiotic species to the base, sugar, or phosphate portions of the DNA molecule and are widely believed to play a role in the process of carcinogenesis. Various polycyclic aromatic hydrocarbons have been targeted as compounds which, once metabolized, possess the ability to bind to DNA. The techniques normally employed to characterize these species at physiologically significant levels, including ${ }^{32}$ P-postlabeling $[1,2]$, immunoassay $[3,4]$, and fluorescence spectroscopy $[5,6]$, are generally not able to provide pertinent structural information for identification of unknowns. Although the application of mass spectrometry to the structural analysis and detection of polyaromatic hydrocarbon ( $\mathrm{PAH}$-DNA adducts has been somewhat limited, notable contributions to this field of study have been made by a number of groups [7-18]. However, except in a few cases [12-14], little attempt has been made to develop mass spectrometric techniques for the characterization of nanogram or lower amounts of the intact PAH adducts.

Address reprint requests to Paul Vouros, Department of Chemistry, Northeastern University, Baston, MA, 02115.
Polycyclic aromatic hydrocarbons are ubiquitous in the environment and arise from various sources including industrial processes and incomplete combustion of such organics as fossil fuels, foods, and tobacco. Once human or animal exposure to these species has occurred, some form of metabolic activation generally converts these PAHs to reactive electrophiles which are able to bind to DNA [19]. In cases where adduct formation is studied via in vitro reactions of carcinogen metabolites with DNA or via in vivo studies in which animals are dosed with carcinogens, methods which provide tor the separation and identification of a limited number of adducts at the level of one adduct in $10^{3}-10^{7}$ normal nucleotides are required. When adducts in human tissues are studied [20], the analysis may be more difficult as complex mixtures of adducts may be present at very low levels (e.g., one adduct in $10^{8}-10^{10}$ normal nucleotides).

Continuous flow-fast atom bombardment (CF-FAB) has proven to be a useful technique for the low-level detection of FAB-amenable analytes and has been found to lend itself well to interfacing with various separation methods [21-23]. With regard to the combination of capillary zone electrophoresis (CZE) with 
CF-FAB, excellent performance has been realized for both liquid junction $[24,25]$ and coaxial flow $[26,27]$ interfaces.

Recently, flow injection CF-FAB was applied to the characterization of deoxynucleoside adducts, with encouraging results [28]. In addition, a CZE method has been developed for the separation and trace level UV detection of DNA adducts isolated from in vitro reaction mixtures [29]. We have explored the utility of CZE coupled with CF-FAB triple quadrupole mass spectrometry for the characterization of DNA adducts with the ultimate goal of analyzing for these compounds in biological matrices.

\section{Experimental}

Chemicals. The structures of the four adducts used in this study are shown in Figure 1. N-acetyl-N(deoxyguanosin-8-yl)-2-aminofluorene was prepared according to the method of Heflich et al. [30, and references therein]. $N^{2}$-(anthracen-9-ylmethyl)-deoxyguanosine was prepared [31] and donated by Dr. L. W. McLaughlin (Boston College). N-(deoxyguanosin8-yl)-2-aminofluorene and $\mathrm{N}$-(deoxyguanosin-8-yl)-4aminobiphenyl were donated by Dr. Frederick Beland (National Center for Toxicological Research). All sol vents were high performance liquid chromatography grade and were purchased from J. T. Baker (Medford, MA) or Fisher (Medford, MA), except for water, which was prepared by the deionization and UV irradiation of house distilled water using a Sybron/Barnstead combined Nanopure II/Organicpure (Boston, MA) system. Glycerol $(99.9 \%)$ was purchased from J. T. Baker. High performance liquid chromatography grade ammonium acetate, ammonium hydroxide, and acetic acid were obtained from Fisher. The acety-
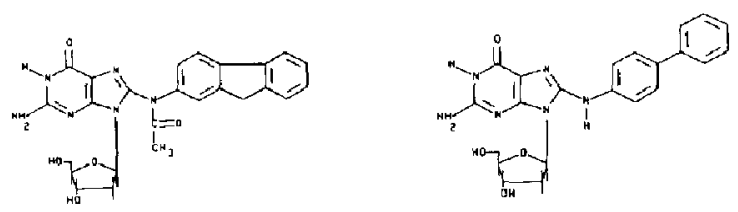

N-acetyl-N-(deoxyguanosi n-8--yi)-2-2 minalluorene

N.(deaxyzanosia-8-yl)-4.anin obiphenyl
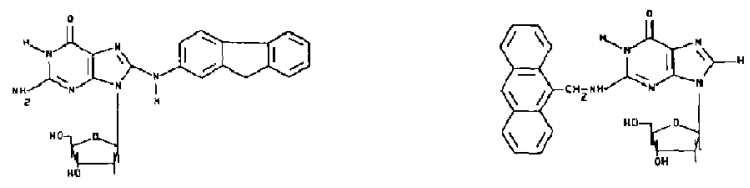

N-(deaxyeus nosin-8-y y)-2-2ntinonworene

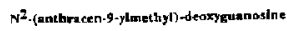

Figure 1. Structures of the PAH and amino-PAH adducts of deoxyguanosine used in the study. laminofluorene and methylanthracene adducts were weighed out on a microbalance and dissolved in methanol, while the aminobiphenyl adduct concentration in methanol was determined by UV absorbance at $305 \mathrm{~nm}$ [32]. The aminofluorene adduct solutions used were old and therefore the adduct amounts reported for the various analyses represent estimated values.

Instrumentation and Conditions. The CZE system employed a power supply manufactured by Glassman High Voltage, Inc. (Whitehouse Station, NJ) and was adjustable up to $30 \mathrm{kV}$. Separations were carried out at $20 \mathrm{kV}(14 \mu \mathrm{A})$ employing a $70 \mathrm{~cm} \times 75 \mu \mathrm{m}$ i.d. polyimide-coated fused silica capillary purchased from l'olymicro l'echnologies (Phoenix, AZ). 'I'he ('ZE' buffer consisted of a $0.01 \mathrm{M}$ solution of $\mathrm{NH}_{4} \mathrm{OAc}$ in water adjusted to $\mathrm{pH} 9.5$ with $\mathrm{NH}_{4} \mathrm{OH}$. The buffer solution was degassed by sonication under vacuum and was filtered daily by using a luer lok $10 \mathrm{ml}$ B-D syringe fitted with a $0.2 \mu \mathrm{m}$ Gelman Acrodisc CR disposable filter cartridge obtained from Fisher. Injections were made by gravity siphoning of the sample solution into the CZE capillary by raising the anode end of the capillary to a level $5 \mathrm{~cm}$ higher than the cathode end. The amount sampled into the capillary was determined by allowing an aliquot of CZE buffer to be siphoned for $\sim 30$ minutes and then subtracting the weight of the sample vial after siphoning from that measured initially. Taking the buffer density to be 1 $\mu \mathrm{g} / \mathrm{nL}$ and assuming that the volume of the buffer would equal the amount of sample solution siphoned per unit time gave the sample injection volume. The amount of buffer siphoned per unit time was found to be $28.9 \mathrm{~nL} / \mathrm{min}(\mathrm{SD}=2.6, \mathrm{n}=3)$ and 5 -second siphons were used for all sample injections.

A VG Quattro triple quadrupole mass spectrometer (VG Instruments, Manchester, UK), outfitted with a cesium ion gun operated at $9 \mathrm{kV}$, was employed for all mass spectrometric analyses. Full scan spectra were acquired over the $m / z 100-600$ range at a rate of 1 $\mathrm{s}$ /scan, while constant neutral loss (CNL) spectra were acquired over the $m / z 250-600$ range at $2.5 \mathrm{~s} / \mathrm{scan}$. Multiple reaction monitoring (MRM) studies, employing a peak top dwell time of $1 \mathrm{~s} /$ reaction, were conducted by sequentially monitoring the loss of deoxyribose (116 u) from the $\mathrm{MH}^{+}$of each of the four adducts in the mixture. For the CNL and MRM analyses, a collision energy of $70 \mathrm{eV}$ was employed and argon was used as the collision gas. The collision gas pressure was adjusted to a level sufficient to attenuate the $m / z 373$ (aglycone) ion of the acetylaminofluorene adduct to $30 \%$. The dynamic FAB matrix, which consisted of $1 \%$ glycerol, $5 \%$ acetonitrile, and $19 \%$ methanol in water, was delivered to the mass spectrometer through the CF-FAB probe via a $45 \mathrm{~cm} \times$ $50 \mu \mathrm{m}$ i.d. polyimide-coated fused silica capillary obtained from Polymicro Technologies. The CF-FAB solution was degassed and filtered in the same fashion as that employed for the CZE buffer. 
Capillary Zone Electrophoresis/Mass Spectrometry Interface. The interface used for this work is pictured in Figure 2. While it is similar in certain respects to liquid junction interfaces previously described in the literature (for a review of advances in this field, see [23], chapter $\mathbf{6}_{r}$ and references therein), it contains several unique features. First, two $1 / 8^{\prime \prime}$ Nalgene tees (Plainfield, NJ) were employed in an attempt to keep bubbles which form at the cathode from entering the mass spectrometer. Second, a $20-u L$ thick walled glass blood capillary, flame drawn to a suitable i.d., joined the two tees and provided a snug alignment for the $\mathrm{C} \angle \mathrm{E}$ and CF-FAB capillaries. The i.d. of the glass capillary was just wide enough to allow for the free flow of CF-FAB solution around the CZE capillary which was then vacuum drawn (along with the CZE eluent) at $\sim 2$ $\mathrm{uL} / \mathrm{min}$ into the mass spectrometer. The CF-FAB matrix solution was contained in a reservoir mounted at a height just above the level of the interface to allow a low gravity-induced flow to continually replenish the supply of CF-FAB matrix solution in the interface. The cathode tee was left unsealed on the CF-FAB capillary end to avoid pressure build-up in the interface. Fittings were made of small pieces of tubing and Eppendorf pipet tips.

\section{Results and Discussion}

In the structural characterization of deoxynucleoside adducts several types of information are sought, including the identities of the deoxynucleoside and the carcinogen and their point of covalent attachment. When analyzing adducts formed in vivo, the metabolism of the carcinogen prior to adduction to the nucleic acid constituents will be important to establish. For in vivo reactions and reactions of carcinogen metabolites with DNA in vitro, sample preparation techniques may give rise to anomalous adducts which require characterization. For example, several reports have discussed unexpected adduct products, including those resulting from the imidazole ring opening of deoxyguanosine adducts in alkaline media [33], oxida-

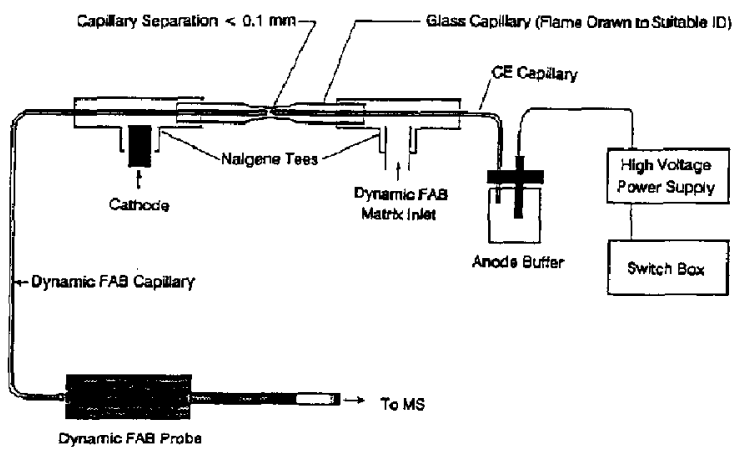

Figure 2. The CZE CF-FAB mass spectrometry system. Certain portions of the system are not drawn to scale with regard to one another. tion of the adenine portion of adenosine adducts possibly due to the presence of deaminase impurities in alkaline phosphatase [34], and the incomplete digestion of adducted nucleotides [35]. The results below illustrate the manner in which a routine separation/ mass spectrometry method for the identification/ detection of adducts at the nanogram to picogram level is able to contribute to this field of research.

rigure 3a shows the CZE/UV electropherogram obtained when a $1.3 \mathrm{ng}$ injection of the acetylaminofluorene adduct was made. The mass chromatogram of $m / z 373$ is given in the inset. In both cases, the $w_{1 / 2}$ was approximately 8 seconds. It was typically found that the interface made a minimal contribution to band broadening, although significant tailing of peaks (possibly occurring at the CF-FAB probe tip) was often observed. It is not clear why the broadening due to the interface was not more severe. Because this efficiency was considered suitable given the scan rate of the mass spectrometer (especially for the acquisition of collision-induced dissociation data
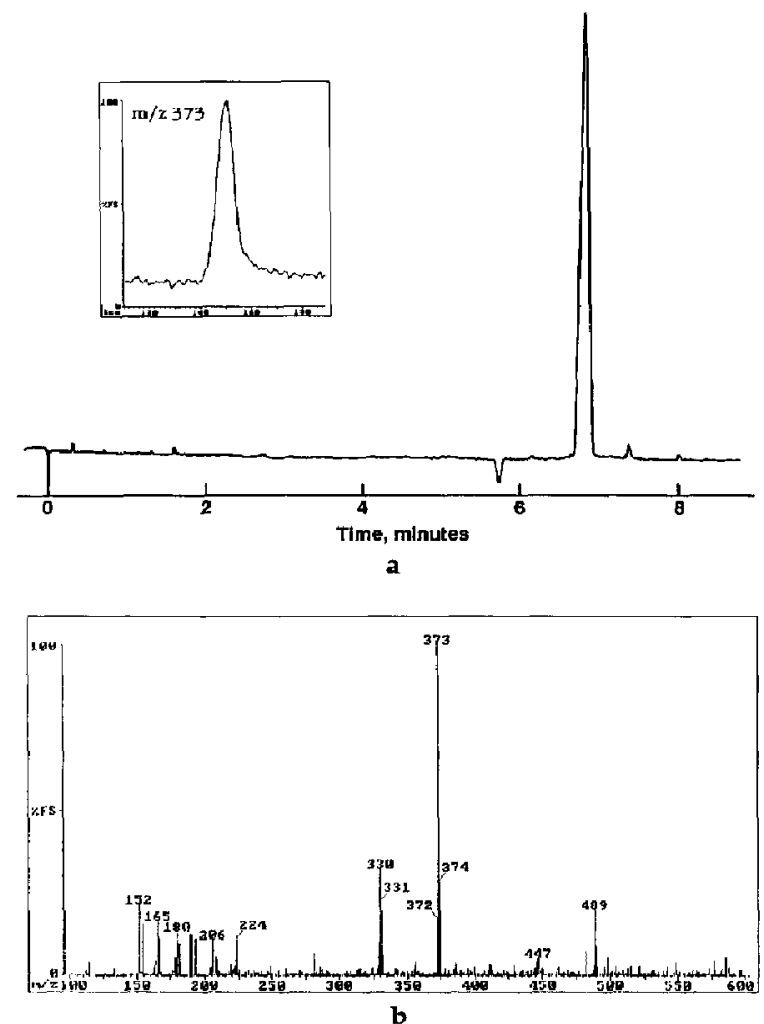

Figure 3. (a) CZE-UV electropherogram obtained for a $1.3 \mathrm{ng}$ injection of the acetylaminofluorene adduct. UV detection at $279 \mathrm{~nm}$. Inset: Ion trace of $\mathrm{m} / \mathrm{z} 373$ for a $1.3 \mathrm{ng}$ injection of the adduct through the interface. $0.01 \mathrm{M} \mathrm{NH}_{4} \mathrm{OAc}$ buffer adjusted to pH 9.5 with $\mathrm{NH}_{4} \mathrm{OH}$. (b) The full scan spectrum obtained from summation of the most intense scans across the peak with background subtraction. 
where $1 \mathrm{~s} / 150$ mass-to-charge ratio units was required for the collection of continuum data), no further attempt was made to optimize the interface. Figure $3 \mathbf{b}$ shows the full scan spectrum obtained from the summation of the most intense scans across the $m / z 373$ peak in Figure 3a with background subtraction. The spectrum shows the characteristic $\mathrm{MH}^{+}$and $\mathrm{AH}_{2}^{+}$(loss of deoxyribose) ions as well as ions due to the loss of the acetyl group from these species $(m / z 446 / 447$ and $330 / 331$, respectively).

Ions appearing in the $m / z 150-225$ range provide information about the PAH and deoxynucleoside identities and about their site of covalent linkage. Because spectral summation and background subtraction for low-intensity ions sometimes gives rise to artifacts in the spectrum, reconstructed mass chromatograms were used to verify the presence of ions in the $m / z 150-225$ range. Analyte-related ions which were confirmed by thesc traces were $m / z 152,165,180,206$, and 224; their assignment has been discussed in detail previously [12]. Briefly, $m / z 224$ corresponds to the protonated acetylaminofluorene species while $m / z 165$ and 180 represent fragments thereof, while $m / z 152$ likely corresponds to protonated guanine. The ion appearing at $m / z 206$ arises from cleavage through the imidazole ring of guanine to give a fragment consisting of aminofluorene plus $\mathrm{CN}$ from the guanine base. This ion is significant in that it suggests the purine imidazole ring as the site at which acetylaminofluorene is adducted to the deoxyguanosine.

Figure 4 shows that constant neutral loss scanning of $116 \mathrm{u}$, corresponding to the loss of deoxyribose, provides strong signals for the $\mathrm{MH}^{+}$ions of $1.3 \mathrm{ng}$ each of the four adducts injected as a mixture. Figure 4a shows the constant neutral loss spectrum which results from summation of the entire set of scans acquired in the CZE/MS run. The data were acquired in continuum mode and the summed spectra were smoothed and centroided. This technique constitutes a

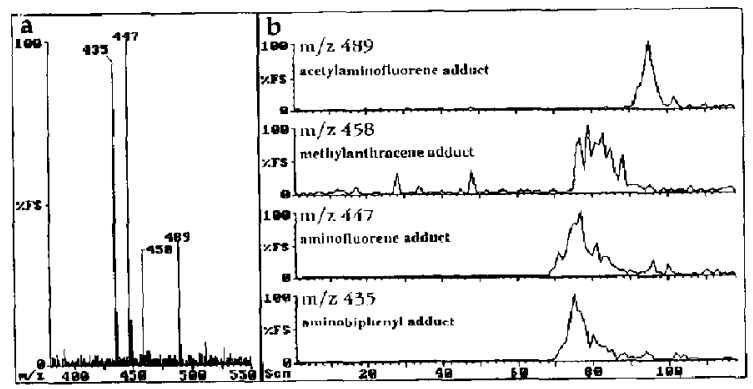

Figure 4. (a) The CNL spectrum which resulted from the summation of the entire set of continuum scans acquired in the CZE/CF-F $\Lambda B$ mass spectrometry run followed by smoothing and centroiding. (b) Traces of the $\mathrm{MH}^{+}$species of the four adducts present in the synthetic mixture as identified by constant neutral loss scanning of the loss of deoxyribose (116 u) from $\mathrm{MH}^{+} .1 .3 \mathrm{ng}$ of each adduct was injected. $0.01 \mathrm{M} \mathrm{NH}_{4} \mathrm{OAc}$ buffer adjusted to $\mathrm{pH} 9.5$ with $\mathrm{NH}_{4} \mathrm{OI}$. powerful method for providing molecular weight information for adducts and for screening for normal deoxynucleosides and deoxynucleoside adducts in mixtures. Once the summation spectrum has targeted possible adduct molecular weights, tracing of these ions will eliminate the potential misinterpretation of a signal resulting from an interfering background ion or an adduct that gives rise to more than one loss. For instance, in addition to the four $\mathrm{MH}^{+}$species, the $\left[\mathrm{M}-\mathrm{CH}_{2} \mathrm{CO}\right] \mathrm{H}^{\prime}$ ion of the acetylaminofluorene adduct also gives a loss of $116 \mathrm{u}$. Other types of neutral losses may also be useful for the detection of various types of adducts. For example, the methylanthracene adduct gives a very strong $m / z 191$ signal, corresponding to [anthracene- $\left.\mathrm{CH}_{2}\right]^{\prime}$ in its normal scan spectrum. This fragment represents a loss of the deoxynucleoside from the $\mathrm{MH}^{+}$ion, which may be a useful transition to monitor for this class of adducts.

Multiple reaction monitoring of the loss of denxyribose from DNA adducts provides a useful method for the low-level detection of target adducts. Figure 5 shows the sequential monitoring of the loss of deoxyribose from the protonated molecular species of $63 \mathrm{pg}$ of each of four adducts. In this case, a pH 3.7 buffer was used and was found to provide a good separation of the C8 adducts. This is presumably due to the fact that, at the lower $\mathrm{pH}$, separability was based upon protonation at the $\mathrm{N} 7$ position $\left(\mathrm{pK}_{\mathrm{a}}=3.3\right.$ in deoxyguanosine), which for adducts of similar size and composition provided an enhanced selectivity over separations carried out at $\mathrm{pH} 9.5$ (corresponding to dissociation at $\mathrm{N} 1, \mathrm{pK}_{\mathrm{a}}=9.4$ in deoxyguanosine). The acquisition was started 3 minutes after mixture injection.

The CZE conditions employed for this study were initially developed for the separation of DNA adducts from unmodified nucleosides and nucleotides, salts, proteins, and other impurities which might be present in a biological system [29]. $\Lambda$ s adduct standards are not widely available for the development of analytical methodology, we anticipate that fine tuning of the CZE conditions will be required for each future system studied.

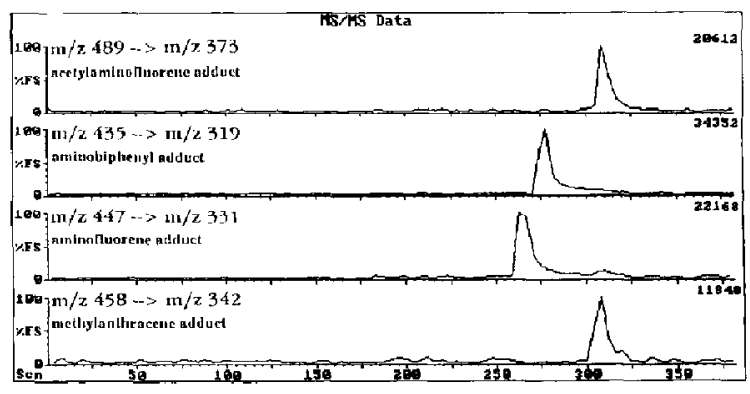

Figure 5. Traces of the four MRM signals arising from the sequential monitoring of the loss of deoxyribose from the $\mathrm{MH}^{+}$ ion of $63 \mathrm{pg}$ of each of the four adducts in the injected mixture. $0.01 \mathrm{M} \mathrm{NH}_{4}$ OAc buffer adjusted to $\mathrm{pH} 3.7$ with acetic acid. 


\section{Conclusions}

The data shown demonstrate the utility of the $\mathrm{CE}$ CFFAB mass spectrometry technique for the low-level characterization of standard adduct mixtures. Tandem mass spectrometry is capable of providing low nanogram detection of unknown adducts by constant neutral loss scanning, while multiple reaction monitoring dala show low picogram limits for the detection of target adducts. (Unfortunately, given the small injection volumes typically required for good CZE performance, the picogram detection limit achieved in the MRM study corresponds to a concentration detection limit in the mid $10^{-5} \mathrm{M}$ range. We are currently considering the adaptation of techniques which will allow for larger volumes of sample to be injected onto the CZE column for this application.) The use of an on-line separation/mass spectrometry systen will allow for the detection of trace amounts of DNA adducts in mixtures which may be difficult to isolate and collect off-line. The results of this study suggest the feasibility of conducting these analyses at physiologically significant levels in biological matrices.

\section{Acknowledgments}

This work was supported by EPA Cooperative Agreement CR815135, an American Chemical Society summer fellowship sponsored by the Suciety of Analytical Chemists of Pittsburgh, and a fellowship from the Arthur D. Little Foundation. Financial support by NIH through an instrument grant (\#1S10RR05602-01) is also gratefully acknowledged. We thank Mohamed Itani for synthesizing the acetylaminofluorene adduct. This is publication \#509 from the Barnett Institute.

\section{References}

1. Randerath, K.j Reddy, M. V.; Gupta, R. C. Proc. Nati. Acad. Sci. USA 1981, 78, 6126-6129.

2. Gupta, R. C.; Reddy, M. V.; Randerath, K. Carcinogenesis 1982, 3, 1081-1092.

3. Harris, C. C.; Yolken, R. H.; Hsu, I.-C. Methods Cancer Res. $1982,20,213-243$.

4. Roberts, D. W.; Benson, R. W.; Groopman, J. D.; Flammang, T. J.; Nagle, W. A.; Moss, A. J.; Kadlubar, F. F. Cancer Res. 1988, 48, 6336-6342.

5 Vahakangas, K; Trivers, G; Rowe, M: Harris, C. C. Enz1. Health Persp. 1985, 62, 101-104.

6. Jankowiak, R.; Small, G. J. Anal. Chem. 1989, 61, $1023 \mathrm{~A}-1032 \mathrm{~A}$.

7. Dietrich, A. M; Guenat, C. R.; Tomer, K. B; Ball, L. M Carcinogenesis 1988, $9,2113-2119$.

8. Rogan, E. C.; Cavalieri, E. L.; Tibbels, S. R.; Cremonesi, P.; Warner, C. D.; Nagel, D. L;; Tomer, K. B,; Cerny, R. L.; Gross, M. L. J. Am. Chem. Soc. 1988, 110, 4023-4029.
9. Dino, J. J.; Guenat, C. R.; Tomer, K. B.; Kaufman, D. G. Rapid Comm. Mass Spec. 1987, 1, 69-71.

10. Tomer, K. B.; Gross, M. L.; Deinzer, M. L. Anal. Chem. 1986, $58,2527-2534$.

11. Tondeur, Y.; Moschel, R. C.; Dipple, A.; Koepke, S. R Anal. Chem, 1986, 58, 1316-1324

12. Annan, R. S.; Giese, R. W.; Vouros, P. Anal. Biochem. 1990, 191, 86-95.

13. Pavlovich, J. G.; Barofsky, E.; Jiang, L. F.; Barofsky, D. F. proceedings of the 38th Annual ASMS Conference on Mass Spectrometry and Applied Topics; Nashville, TN, 1991; pp 80-81.

14. Chiarelli, M. P.; Bryant, M. S; Lay, J. O. Proceedings of the 38th Annual ASMS Confercnce on Mass Spcctrometry and Allied Topics; Nashville, TN, 1991; pp 967-968.

15. Pogracz, K.; Kaur, S.; Burlingame, A. L.; Bodell, W. J. Carcinogenesis 1989, 10, 1009-1013.

16. Fu, P. P.; Miller, D. W.; Von Tungeln, L. S.; Bryant, M. S.; Lay, J. O.; Huang, K.; Jones, L.; Evans, F. E. Carcinogenesis 1991, 12, 609-616.

17. Mitchum, R. K.; Evans, F. E.; Freeman, J. P.; Roach, D. int. J. Mass Spectrom. Ion Proc. 1983, 46, 383-386.

18. Dolnikowski, G. G.; Gross, M. L,; Cavalieri, E. L. J. Am. Soc. Mass Spectrom. 1991, 2, 256258.

19. Phillips, D. H. In The Molecular Basis of Cancer; Farmer, P. B.; Walker, J. M., Eds.; Wiley-Interscience: New York, 1985; PP 133-179.

20. Chacko, M.; Gupta, R. C. Carcinogenesis 1988, 9, 2309-2313.

21. Ito, Y.; Takeuchi, T.; Ishii, D.; Goto, M. J. Chrom. 1985, 346, 161-166.

22. Caprioli, R. M.; Fan, T.; Cottrell, J. S. Anat. Chem. 1986, 58, 2949-2954.

23. Continuous-flow Fast Atom Bombardment Mass Spectrometry; Caprioli, R. M., Ed.; John Wiley; New York, 1990.

24. Reinhoud, N. J.; Niessen, W. M. A.; Tjaden, U. R.; Gramberg, L. G.; Verheij, E. R.; van der Greef, J. Rapid Comm. Mass Spec. 1989, 3, 348-351.

25. Caprioli, R. M.; Moore, W. T.; Martin, M.; DaGue, B. B.; Wilson, K.; Moring, S. J. Chrom. 1989, 480, 247-257.

26. Mosley, M. A.; Deterding, L. J.; Tomer, K. B.; Jorgenson, J. W. I. Chrom. 1989, 480, 197-209.

27. Mosely, M. A.; Deterding, L. J.; Tomer, K. B.; Jorgenson, J. W. Anal. Chem. 1991, 63, 109-114.

28. Wolf, S. M.; Annan, R. S.; Giese, R. G.; Vouros, P. Biol. Mass Spectrom., accepted.

29. Jackim, E.; Norwood, C. J. High Res. Chrom. 1990, 13, 195-196.

30. Heflich, R. H.; Djuric, Z.; Zhuo, Z.; Fullerton, N. F.; Casciano, D. A.; Beland, F. A. Enviton. Molec. Mutagen. 1988, 11, 167-181.

31. Casale, R.; McLaughlin, L. W. J. Am. Chem. Soc. 1990, 112, 5264-5271.

32. Shapiro, R.; Underwood, G. R.; Zawadzka, H.; Broyde, S.; Hingerty, B. E. Biochemistry 1986, 25, 2198-2205.

33. Kriek, E.; Westra, J. G. Carcinogenesis 1980, 1, 459-468.

34. Declos, K. B.; Miller, D. W.; Lay, J. O.; Casciano, D. A.; Walker, R. P.; Fu, P. P.; Kadlubar, F. F. Carcinogenesis 1987, 8, 1703-1709.

35. Gupta, R. C. Proc. Natl. Acad. Sci. 1984, 81, 6943-6947. 